\title{
An Adaptive Sparse Sampling Scheme for Scanning Electron Microscopy using Delauney Triangulation
}

\author{
Tim Dahmen ${ }^{1, *}$, Patrick Trampert ${ }^{1,2}$ \\ 1. German Research Center for Artificial Intelligence (DFKI), Saarbrücken, Germany. \\ 2. Saarland University, Saarbrücken, Germany \\ * Corresponding author: Tim.Dahmen@dfki.de
}

Scanning Electron Microscopy (SEM) is a widely used microscopy technique with applications in fields such as material science, life science, and semiconductor research. Due to the large number of pixels in a dataset, the pixel-dwell time, which is the amount of time the electron beam illuminates a single pixel, is a crucial parameter for the aforementioned techniques. The pixel-dwell time required for a sufficiently high SNR leads to large data acquisition times, in particular for 3D techniques.

Often, scanning the sample on a regular grid is not the optimal sampling strategy and alternative strategies have been proposed [1]. For samples with a sparse gradient (for example material science samples with homogeneous phases and sharp phase boundaries), it is desirable to concentrate the largest amount of dose on the phase boundaries, where the image contains the highest spatial frequencies. One way to obtain such a dose concentration is to scan the image twice [2]. An initial, noisy scan allows to identify non-zero gradients in the image. A second, sparse scan images only these areas with high details. Both scans are then combined to form the image.

We now present an improved version of this work that eliminates the need for an initial dense scan of the image. Instead, we present an iterative sampling scheme that uses a sequence of sparse scans to gather information about the sample. The decision which pixels will be scanned depends on the results of the previous scans. The sampling is initialized by a stratified random scan where one randomly selected pixel out of every square of $3 \times 3$ pixels is scanned (Figure 1a). A Delauney triangulation is computed to determine neighbourhood relations between scanned pixels. In this scheme, a Voronoy diagram is computed with the scanned pixel positions as seeds. The triangulation contains an edge between two scanned pixels if the corresponding Voronoy cells share a border (Figure 1b). The edges are then used to make a decision which pixels are scanned in the next iteration. If the pixels at the end of the edge differ too much, the edge is subdivided, and an additional pixel is scanned in the middle of the edge (Figure 1c). Using this method, the sample is scanned repeatedly, where every iteration visits one additional percent of the pixels of the image. After nine iterations, a total of approximately $20 \%$ of all pixels have been scanned. The final image scan be obtained from the sparse dataset using natural neighbour interpolation (Figure 2). Better reconstruction might be possible using dictionary based methods [3], of these techniques can be adapted to handle heterogeneous sampling densities.

\section{References:}

[1] S Grosche et al. IEEE International Conference on Imaging Systems and Techniques (2018) p. 1-6.

[2] T Dahmen et al. Scientific Reports 6 (2016) 25350.

[3] F Boughorbel et al. Microsc. \& Microanal. 23 S1 (2017) p. 150-151. 
a

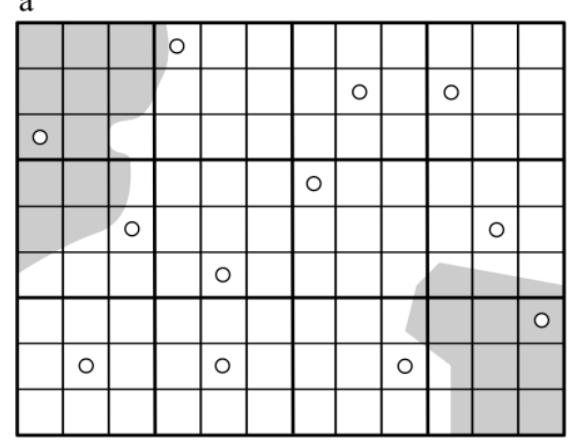

b

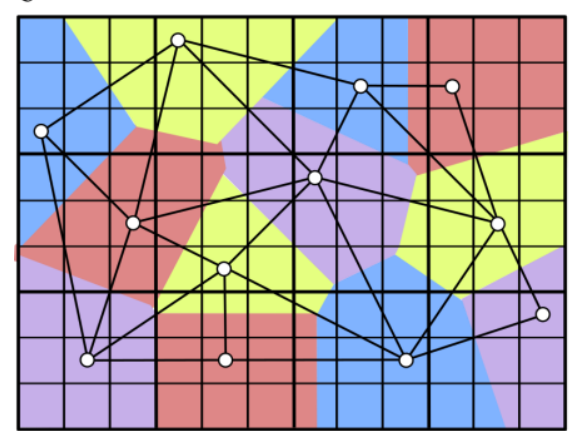

c

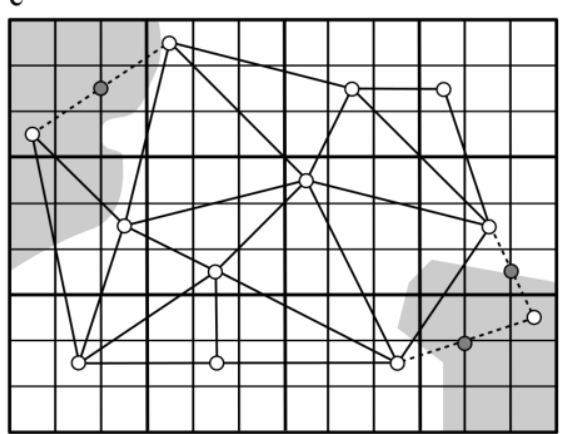

d

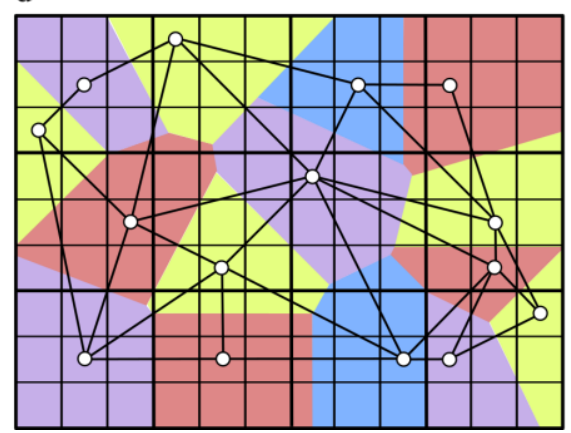

e

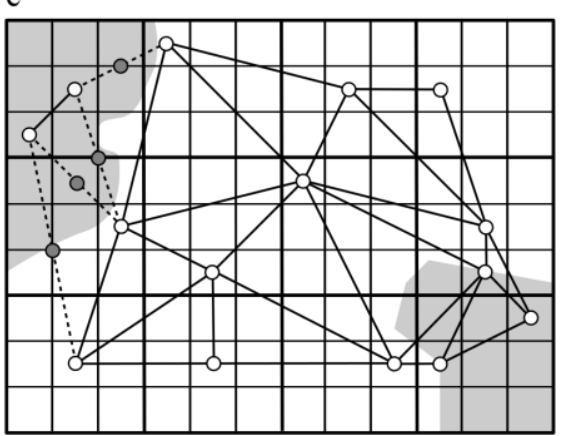

f

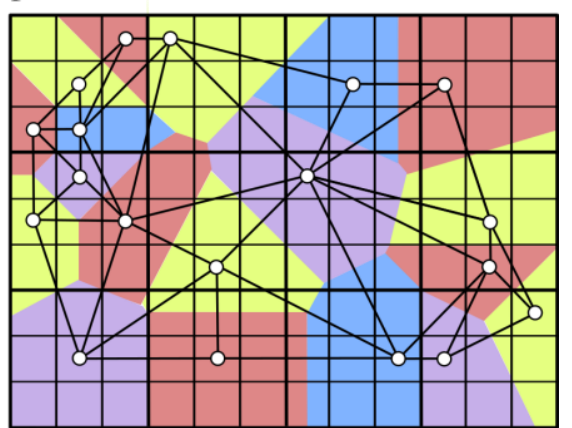

Figure 1. a) The scanning process is initialized by $3 \times 3$ stratified random sampling. In this technique, one pixel out of every square of $3 \times 3$ pixels is scanned. b) The neighborhood relation of scanned pixels is determined by means of Delauney triangulation. Hereby, a Voronoy diagram is computed with the scanned pixel positions as seeds. Two pixels are connected by an edge if the corresponding Voronoy cells share a border. c) If the gray values of two pixels that are connected by an edge differ too much, the edge is subdivided and an pixel in the middle of the edge is scanned. d) The Delauney triangulation is then updated incrementally. e,f) The procedure iterates.

a

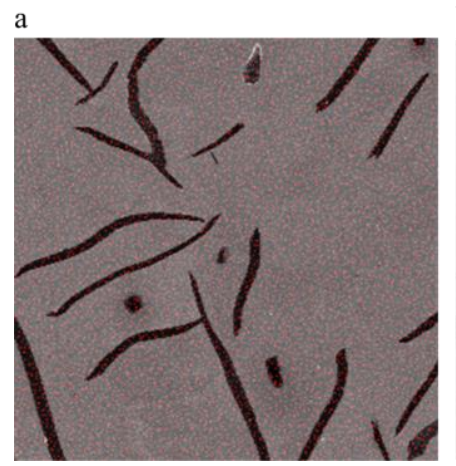

$\mathrm{b}$

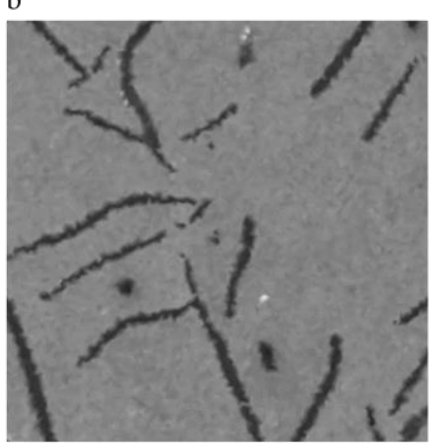

$\mathrm{c}$

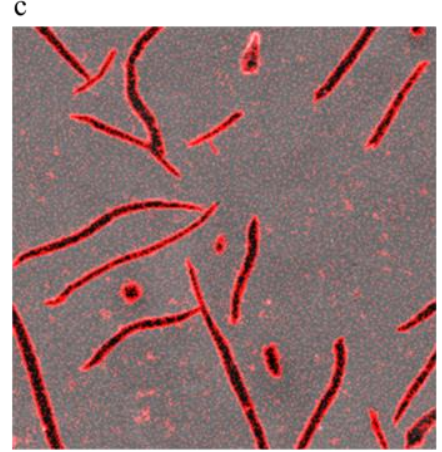

$\mathrm{d}$

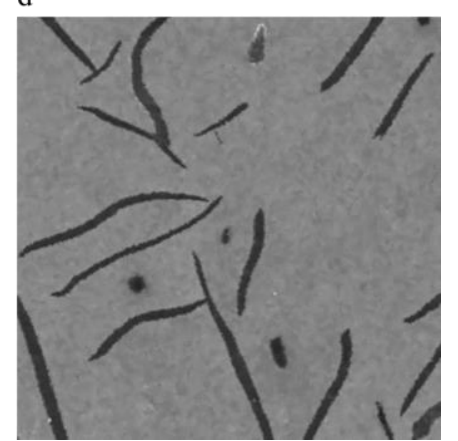

Figure 2. Image of a cast iron sample with lamella graphite inclusions. a) The scanning is initialized by scanning approximately $11 \%$ of the sample (red pixels). b) A natural neighbor interpolation of the data set after initialization. c) After 9 additional iterations, approximately $20 \%$ of the pixels have been scanned (red pixels). d) A natural neighbor interpolation of the data set after 10 iterations. 